\title{
A COROLLARY OF ALEXANDER'S INEQUALITY
}

\section{TAKAHIKO NAKAZI}

Abstract. A simple corollary of Alexander's inequality is shown for a uniform algebra. As a result, we give an inequality between the $L^{\infty}$-type norm of a given analytic function and the $L^{1}$-type norm of the derivative.

Mathematics subject classification (2010): 46J10, 30D55.

Keywords and phrases: Alexander's inequality, Hardy space, uniform algebra.

\section{REFERENCES}

[1] H. Alexander, Projections of polynomial hulls, J. Funct. Anal., 13 (1973), 13-19.

[2] M. Chō AND T. NAKAZI, Spectral area estimates for norms of commutators, J. Korean Math. Soc., 44 (2007), 855-861.

[3] P. Duren, Theory of $H^{p}$ Spaces, Academic Press, New York, 1970.

[4] T. Gamelin, Uniform Algebras, Prentice-Hall, Englewood Cliffs, N. J., 1969.

[5] T. Gamelin, On an estimate of Axler and Shapiro, Math. Ann., 272 (1985), 189-196.

[6] T. GAMELIN AND D. KHAVINSON, The isoperimetric inequality and rational approxination, Amer. Math. Month., 96 (1989), 18-30.

[7] D. Khavinson, A note on Toeplitz operators, "Banach Spaces", 89-94, Lecture Notes in Math., 1166, Springer, Berlin, 1985.

[8] T. NAKAZI, Complete spectral area estimates and self-commutators, Michigan Math. J., 35 (1988), 435-441. 